\title{
A New Class of Production Function
}

\author{
D. Bayanjargal1', B. Yerkyebulan², Ts. Battsukh³ \\ ${ }^{1}$ National University of Mongolia, Ulaanbaatar, Mongolia \\ ${ }^{2}$ The Shildeg American Mongolian School, Ulaanbaatar, Mongolia \\ ${ }^{3}$ University of Finance and Economics, Ulaanbaatar, Mongolia \\ Email: bayanjargal@seas.num.edu.mn,yerkyebulan.b@gmail.com, batsukh.ts@ufe.edu.mn
}

How to cite this paper: Bayanjargal, D., Yerkyebulan, B., \& Battsukh, T. (2020). A New Class of Production Function. Theoretical Economics Letters, 10, 356-365. https://doi.org/10.4236/tel.2020.102024

Received: March 10, 2020

Accepted: April 20, 2020

Published: April 23, 2020

Copyright $\odot 2020$ by author(s) and Scientific Research Publishing Inc. This work is licensed under the Creative Commons Attribution International License (CC BY 4.0).

http://creativecommons.org/licenses/by/4.0/

\section{(c) (i) Open Access}

\begin{abstract}
In this article, we propose a new class of production functions in the new classical theory of economics and study its qualities based on Euler's relation of quasi-homogeneous functions. In a market economy environment, it is crucial to establish a firm's profitability and draw conclusions about the operation and to make a variety of assumptions using a function that is more consistent with reality in the future. We showed that the quasi-homogeneous function is a general form of the well known production functions such as Cobb-Douglas and Constant Elasticity of Substitution (CES). We have made some qualitative and quantitative analysis and compare our results with the classical models using statistical data of Japan.
\end{abstract}

\section{Keywords}

Euler's Theorem, Quasi-Homogeneous, Production Function

\section{Quasi-Homogeneous Production Functions and Its Properties}

The production function is one of the key concepts of mainstream neoclassical theories. By assuming that the maximum output technologically possible from a given set of inputs is achieved, economists use a production function in analysis, representing the mathematical formalization of the relationship between production and the factors that actually contribute to that production. Such a function is a mapping

$$
f: R_{+}^{n} \rightarrow R_{+}, n \geq 2 \text {, given by } f\left(x_{1}, x_{2}, x_{3}, \cdots, x_{n}\right)
$$

where $n$ denotes the number of factors of production (inputs), $x_{1}, x_{2}, \cdots, x_{n}$ are the inputs and $f$ is the level of output (production).

Definition 1 (Alina-Daniela \& Gabriel-Eduard, 2019) Let $f$ be a differentiable 
function of $n$ variables $x_{1}, x_{2}, \cdots, x_{n}$ defined on the open set $M\left(M \subset R^{n}\right)$ then $f(M, 0) \rightarrow(R, 0)$ is said to be a quasi-homogeneous function if the following condition holds

$$
\lambda^{\gamma} f\left(x_{1}, x_{2}, x_{3}, \cdots, x_{n}\right)=f\left(\lambda^{\alpha_{1}} x_{1}, \lambda^{\alpha_{2}} x_{2}, \cdots, \lambda^{\alpha_{n}} x_{n}\right)
$$

of degree $\gamma$ with weight vector $\alpha=\left(\alpha_{1}, \alpha_{2}, \alpha_{3}, \cdots, \alpha_{n}\right)$ and all $\lambda>0$. For instance, the production function defined by $f(x, y)=x^{3}+y^{2}$ is a quasi-homogeneous production function of degree 1 with weight vector $\left(\frac{1}{3}, \frac{1}{2}\right)$.

Theorem 1 (Alina-Daniela \& Gabriel-Eduard, 2019) Let $f$ be a differentiable function of $n$ variables $x_{1}, x_{2}, \cdots, x_{n}$ defined on the open set $M\left(M \subset R^{n}\right)$ of degree $\gamma$ with weight vector $\alpha=\left(\alpha_{1}, \alpha_{2}, \alpha_{3}, \cdots, \alpha_{n}\right)$ and all $\lambda>0$. then $f(M, 0) \rightarrow(R, 0)$ is satisfy the generalized Euler identity:

$$
\alpha_{1} \frac{\partial f}{\partial x_{1}} x_{1}+\alpha_{2} \frac{\partial f}{\partial x_{2}} x_{2}+\cdots+\alpha_{n} \frac{\partial f}{\partial x_{n}} x_{n}=\gamma f
$$

Let point $M_{0}\left(x_{1}^{0}, x_{2}^{0}, \cdots, x_{n}^{0}\right)$ is chosen from the set $M$ and the point $N\left(\lambda^{\alpha_{1}} x_{1}^{0}, \lambda^{\alpha_{2}} x_{2}^{0}, \cdots, \lambda^{\alpha_{n}} x_{n}^{0}\right) \subset M, \forall \lambda>0$.

Proof of Theorem 1 Let us set down composite function $y=f\left(x_{1}, \cdots, x_{n}\right)$, $x_{i}=\lambda^{\alpha_{i}} x_{i}^{0}, i=\overline{1, n}$.

Functions $x_{i}(i=\overline{1, n})$ and $f$ are differentiable where the $\lambda=1$ then the $\mathrm{f}$ can be differentiable where the point $M_{0}$. We recall

$$
\frac{\partial x_{i}}{\partial \lambda}=\alpha_{i} \lambda^{\alpha_{i}-1} x_{i}^{0}, \quad(i=\overline{1, n})
$$

and solve the $\frac{\mathrm{d} y}{\mathrm{~d} \lambda}$ where $\lambda=1$, then

$$
\frac{\mathrm{d} y}{\mathrm{~d} \lambda}=\alpha_{1} \frac{\partial f}{\partial x_{1}} x_{1}^{0}+\alpha_{2} \frac{\partial f}{\partial x_{2}} x_{2}^{0}+\cdots+\alpha_{n} \frac{\partial f}{\partial x_{n}} x_{n}^{0}
$$

on the other hand $y=\lambda^{\gamma} f\left(x_{1}^{0}, x_{2}^{0}, \cdots, x_{n}^{0}\right)$, whereas

$$
\frac{\mathrm{d} y}{\mathrm{~d} \lambda}=\gamma \lambda^{\gamma-1} f\left(x_{1}^{0}, x_{2}^{0}, \cdots, x_{n}^{0}\right)=\gamma f\left(x_{1}^{0}, x_{2}^{0}, \cdots, x_{n}^{0}\right)
$$

where the $\lambda=1$ then

$$
\gamma f=\alpha_{1} \frac{\partial f}{\partial x_{1}} x_{1}^{0}+\alpha_{2} \frac{\partial f}{\partial x_{2}} x_{2}^{0}+\cdots+\alpha_{n} \frac{\partial f}{\partial x_{n}} x_{n}^{0}
$$

$M_{0}$ is the any point selected from $M$.

Theorem 2 If $f$ be a differentiable function of $n$ variables $x_{1}, x_{2}, \cdots, x_{n}$ defined on the open set $M\left(M \subset R^{n}\right)$ of degree $\gamma$ with weight vector $\alpha=\left(\alpha_{1}, \alpha_{2}, \alpha_{3}, \cdots, \alpha_{n}\right)$ and all $\lambda>0$ then by substituting $T\left(x_{1}, x_{2}, \cdots, x_{n}\right)=\left(z^{\alpha_{1}}, z^{\alpha_{2}}, \cdots, z^{\alpha_{n}}\right)$, it can be a quasi-homogeneous function with variables $z=\left(z_{1}, z_{2}, \cdots, z_{n}\right)$.

Proof of Theorem 2 According to the given condition of the function, the following equation must be true

$$
\lambda f=\alpha_{1} \frac{\partial f}{\partial x_{1}} x_{1}+\alpha_{2} \frac{\partial f}{\partial x_{2}} x_{2}+\cdots+\alpha_{n} \frac{\partial f}{\partial x_{n}} x_{n} .
$$


substitution of $f$ function, the equation can be defined as follows

$$
\gamma f\left(x_{1}, x_{2}, x_{3}, \cdots, x_{n}\right)=\gamma f\left(z_{1}^{\alpha_{1}}, z_{2}^{\alpha_{2}}, \cdots, z_{n}^{\alpha_{n}}\right)=\gamma f\left(z_{1}, z_{2}, \cdots, z_{n}\right)
$$

to use the composite-function differential rule then

$$
\frac{\partial f}{\partial x_{i}} x_{i}=\frac{\partial f}{\partial z_{i}} \cdot \frac{\mathrm{d} y}{\mathrm{~d} z_{i}} \cdot x_{i}=\frac{\partial f}{\partial z_{i}} \cdot \frac{1}{\alpha_{i}} \cdot x_{i}^{\frac{1}{\alpha_{i}}-1} \cdot x_{i}=\frac{1}{\alpha_{i}} \cdot \frac{\partial f}{\partial z_{i}} z_{i}, \quad(i=\overline{1, n})
$$

by substitution, we can obtain the following equation

$$
\lambda f\left(z_{1}, z_{2}, \cdots, z_{n}\right)=\frac{\partial f}{\partial z_{1}} z_{1}+\frac{\partial f}{\partial z_{2}} z_{2}+\cdots+\frac{\partial f}{\partial z_{n}} z_{n} .
$$

We recall Euler's theorem, we can prove that $\mathrm{f}$ is quasi-homogeneous function of degree $\gamma$. Now let's construct the general form of the quasi-homogeneous function. The equation that was mentioned theorem 1 , for a $f$ function. If we integrate the Equation (1) and estimate the independent original integrals then we can obtain the solution as follows

$$
F\left(\frac{x_{2}^{\frac{1}{\alpha_{2}}}}{x_{1}^{\frac{1}{\alpha_{1}}}}, \frac{x_{3}^{\frac{1}{\alpha_{3}}}}{x_{1}^{\frac{1}{\alpha_{1}}}}, \cdots \frac{x_{n}^{\frac{1}{\alpha_{n}}}}{x_{1}^{\frac{1}{\alpha_{1}}}}\right) \cdot \frac{y}{x_{1}^{\frac{1}{\alpha_{1}}}}=0
$$

if we substitute $y$ for main variables then

$$
y=f\left(x_{1}, x_{2}, \cdots, x_{n}\right)=x_{1}^{\frac{1}{\alpha_{1}}} \Phi\left(\frac{x_{2}^{\frac{1}{\alpha_{2}}}}{x_{1}^{\frac{1}{\alpha_{1}}}}, \frac{\frac{1}{\alpha_{3}}}{x_{1}^{\frac{1}{\alpha_{1}}}}, \cdots \frac{x_{n}^{\frac{1}{\alpha_{n}}}}{x_{1}^{\frac{1}{\alpha_{1}}}}\right)
$$

(11) are quasi-homogeneous function of $n$ variables $x_{1}, x_{2}, \cdots, x_{n}$, defined on the open set $M\left(M \subset R^{n}\right)$, of exponent degree $\gamma$ will be exhausted.

\section{Proposition}

If $f$ is quasi-homogeneous function of degree $\gamma$. In accordance with the following curve,

$$
(*)=\left\{\begin{array}{c}
x_{1}(\lambda)=\lambda^{\alpha_{1}} x_{1}^{0} \\
x_{2}(\lambda)=\lambda^{\alpha_{2}} x_{2}^{0} \\
\vdots \\
x_{n}(\lambda)=\lambda^{\alpha_{n}} x_{n}^{0}
\end{array}\right.
$$

$f$ is concave when $0<\lambda<1$ and convex when $\lambda>1$.

\section{Proof of Proposition}

From the definition of $f$ function the following condition is satisfied if

$$
f\left(x_{1}, x_{2}, \cdots, x_{n}\right)=\lambda^{\gamma} f\left(x_{1}^{0}, x_{2}^{0}, \cdots, x_{n}^{0}\right)=f\left(\lambda^{\alpha_{1}} x_{1}^{0}, \lambda^{\alpha_{2}} x_{2}^{0}, \cdots, \lambda^{\alpha_{n}} x_{n}^{0}\right)
$$

therefore we can imagine $f\left(x_{1}, x_{2}, \cdots, x_{n}\right)$ is a function defined on $\left({ }^{*}\right)$ as a single variable $\varphi(\lambda)$ as follows

$$
\varphi(\lambda)=\lambda^{\gamma} f\left(x_{1}^{0}, x_{2}^{0}, \cdots, x_{n}^{0}\right)
$$

Differentiating the $\varphi(\lambda)$ function twice, we can obtain the following equa- 
tion

$$
\frac{\mathrm{d}^{2} \varphi}{\mathrm{d} \lambda^{2}}=\gamma(\gamma-1) \lambda^{\gamma-2} f\left(x_{1}^{0}, x_{2}^{0}, \cdots, x_{n}^{0}\right)
$$

if we recall that $f$ is positive then concave when $0<\gamma<1$, and convex when $\gamma>1$.

Remark 1 We normalize that $\gamma$ always 1 , if so function's concave and convex conditions are defined by $\alpha_{i}$. A quasi-homogeneous production function of $n$ variables $x_{1}, x_{2}, \cdots, x_{n}$ defined on the open set $M\left(M \subset R^{n}\right)$ of degree 1 with weight vector $\alpha=\left(\alpha_{1}, \alpha_{2}, \alpha_{3}, \cdots, \alpha_{n}\right)$ is concave when $\alpha_{i} \geq 1,(i=\overline{1, n})$.

\section{Quasi-Concave Functions and Properties}

Definition 2 (Arrow, 1961) $f$ is said to be quasi-convex on $M\left(M \subset R^{n}\right)$ if

$$
f\left(\alpha x_{1}+(1-\alpha) x_{2}\right) \leq \max \left(f\left(x_{1}\right), f\left(x_{2}\right)\right), \quad \forall \alpha \in[0,1]
$$

$f$ is said to be quasi-concave on $M\left(M \subset R^{n}\right)$ if

$$
f\left(\alpha x_{1}+(1-\alpha) x_{2}\right) \geq \min \left(f\left(x_{1}\right), f\left(x_{2}\right)\right)
$$

$f$ is called quasi-linear if it is both quasi-concave and quasi-convex.

If the inequalities are strict, and, $x_{1} \neq x_{2}$, then the functions are called a strict convex and concave functions.

\section{Proof Definition 2}

$f$ is quasi-convex if and only if $-f$ is quasi-concave. Note that

$$
\begin{aligned}
& -\min \left\{f\left(x_{1}\right), f\left(x_{2}\right)\right\}=\max \left\{-f\left(x_{1}\right),-f\left(x_{2}\right)\right\} \\
& -\max \left\{f\left(x_{1}\right), f\left(x_{2}\right)\right\}=\min \left\{-f\left(x_{1}\right),-f\left(x_{2}\right)\right\} .
\end{aligned}
$$

First, suppose that $f$ is quasi-concave. If the upper contour set of $f$

$$
U_{f}(\alpha)=\{x \in M: f(x) \geq \alpha\}
$$

and the lower-contour set of $f$

$$
L_{f}(\alpha)=\{x \in M: f(x) \leq \alpha\}
$$

are convex sets for each $\alpha$. Let $\left(x_{1}, x_{2}\right) \in M$ and $\lambda \in(0,1)$. Assume, without loss of generality, that, $f\left(x_{1}\right) \geq f\left(x_{2}\right)$, letting, $f\left(x_{2}\right)=\alpha$, we have $\left(x_{1}, x_{2}\right) \in U_{f}(\alpha)$. By convexity of $U_{f}(\alpha)$, we have $\lambda x+(1-\lambda x) \in U_{f}(\alpha)$ $\Rightarrow f\left(\lambda x_{1}+(1-\lambda) x_{2}\right) \geq f\left(x_{2}\right) \geq \alpha=\min \left(f\left(x_{1}\right), f\left(x_{2}\right)\right)$.

Conversely, suppose $U_{f}(\alpha)$ is convex and empty or contains only one point, it is clear that it is convex, but suppose it contains at least two points $x_{1}$ and $x_{2}$ then $f\left(x_{1}\right) \geq \alpha$ and $f\left(x_{2}\right) \geq \alpha$, so

$$
\begin{gathered}
\min \left(f\left(x_{1}\right), f\left(x_{2}\right)\right) \geq \alpha \\
f\left(\lambda x_{1}+(1-\lambda) x_{2}\right) \geq \min \left(f\left(x_{1}\right), f\left(x_{2}\right)\right)
\end{gathered}
$$

by hypothesis, and so $\lambda x_{1}+(1-\lambda) x_{2} \in U_{f}(\alpha)$, hence $U_{f}(\alpha)$ is a convex set and $f$ is quasi concave. Quasi-concave function is a generalization of concave 
functions since we can show that the set of all quasi-concave functions contains the set of all concave functions.

Theorem 3 (Mayer, 2007) If $f$ is concave on $M$, it is also quasi concave on $M$. If $f$ is convex on $M$, it is also quasi-convex on $M$.

We prove the first part of the theorem and the second part will be proved analogously.

Proof of Theorem 3 Suppose $\mathrm{f}$ is concave, then, for all $x_{1}, x_{2} \in M$ and $\lambda \in(0,1)$ we have

$$
\begin{aligned}
& f\left(\lambda x_{1}+(1-\lambda) x_{2}\right) \geq \lambda f\left(x_{1}\right)+(1-\lambda) f\left(x_{2}\right) \\
& \geq \lambda \cdot \min \left\{f\left(x_{1}\right), f\left(x_{2}\right)\right\}+(1-\lambda) \min \left\{f\left(x_{1}\right), f\left(x_{2}\right)\right\} \\
& =\min \left\{f\left(x_{1}\right), f\left(x_{2}\right)\right\}, \quad \forall \lambda \in[0,1]
\end{aligned}
$$

Theorem 4 (Intriligator, 2002) let $\mathrm{f}$ be a differentiable function on $M$, where $M\left(M \subset R^{n}\right)$ is convex and open. Then $f$ is quasi-concave on $M$ if and only if $\left(x_{1}, x_{2}\right) \in M$ and $f\left(x_{1}\right) \geq f\left(x_{2}\right) \Rightarrow \nabla f\left(x_{2}\right)\left(x_{1}-x_{2}\right)^{\mathrm{T}} \geq 0$.

Proof of Theorem 4 Suppose first that $f$ is quasi-concave on $M$, then

$$
f\left(x_{1}\right) \geq f\left(x_{2}\right) \Rightarrow f\left(\lambda x_{1}+(1-\lambda) x_{2}\right) \geq f\left(x_{2}\right) \text { for } \lambda \in(0,1)
$$

since $f$ is quasi-concave, we have

$$
f\left(x_{2}+\lambda\left(x_{1}-x_{2}\right)\right)=f\left((1-\lambda) x_{2}+\lambda x_{1}\right) \geq \min \left\{f\left(x_{1}\right), f\left(x_{2}\right)\right\}=f\left(x_{1}\right)
$$

Therefore

$$
\frac{f\left(x_{2}+\lambda\left(x_{1}-x_{2}\right)\right)-f\left(x_{2}\right)}{\lambda} \geq 0
$$

As $\lambda \rightarrow 0^{+}$, that is, taking the limit as $\lambda$ approaches through positive numbers, we obtain $f\left(x_{2}\right)\left(x_{1}-x_{2}\right)^{\mathrm{T}} \geq 0$, proving one part of the result.

Conversely

Assume that for all $x_{1}, x_{2} \in M$ such that $f\left(x_{2}\right) \leq f\left(x_{1}\right)$, we have $f\left(x_{2}\right)\left(x_{1}-x_{2}\right) \geq 0$. Pick any $x_{1}, x_{2} \in M$, and suppose without loss of generality that $f\left(x_{1}\right)=\min \left\{f\left(x_{1}\right), f\left(x_{2}\right)\right\}$, we also have

$$
f\left((1-\lambda) x_{1}+\lambda x_{2}\right) \geq \min \left\{f\left(x_{1}\right), f\left(x_{2}\right)\right\}
$$

establishing the quasi-concavity of $f$.

\section{Production Function and Its Properties}

An economy's output of goods and services depends on its quantity of inputs, called the factors of production, and its ability to turn inputs into outputs. The two most important factors of production are capital and labor, also the technology change alters the production function (Solow, 1956). We consider the functions that satisfy the following conditions (Intriligator, 2002):

1) $y=f(x) \geq 0, x \in X^{+}, X^{+} \subset R^{n+}, R^{n+}=\{x \mid x \geq 0\}$, where $X^{+}$is the domain of $f(x)$ function. 
2) $X^{++}=\left\{x \mid \frac{\partial y}{\partial x_{i}} \geq 0\right\}, X^{++} \subset X^{+}, X^{++} \neq \varnothing, i=\overline{1, n}$, where $X^{++}$is said to be "economic domain" (when the resource's price increase, there is no change on production function on this domain)

3) $X^{+++}=\left\{x \mid \sum_{i=1}^{n} \sum_{j=1}^{n} z \frac{\partial^{2} y}{\partial x_{i} \partial x_{j}} z \leq 0, \forall z\right\}, X^{+++} \subset X^{+}, X^{+++} \neq 0$, this means that the production function is a quasi-concave on the domain.

4) $X_{A}=X^{+++} \cap X^{++} \neq \varnothing$. where $X_{A}$ is said to be the set of profit.

5) The production function is twice differentiable.

6) The production function is a quasi-homogeneous of degree $\gamma$, with weight vector $\alpha=\left(\alpha_{1} \cdots \alpha_{n}\right) \in R^{n}$.

Let's consider production function with two variables. According to the condition (6) that was mentioned above, then the production function has the following general form:

$$
y=f\left(x_{1}, x_{2}\right)=x_{1}^{\frac{\gamma}{\alpha_{1}}} \Phi\left(\frac{x_{2}^{\frac{1}{\alpha_{2}}}}{x_{1}^{\frac{1}{\alpha_{1}}}}\right),
$$

according to the Euler's theorem

$$
\alpha_{1} \frac{\partial f}{\partial x_{1}} x_{1}+\alpha_{2} \frac{\partial f}{\partial x_{2}} x_{2}=\gamma f
$$

if we divide both sides $\gamma$, then by substitution $\alpha_{1}=\frac{\alpha_{1}}{\gamma}, \alpha_{2}=\frac{\alpha_{2}}{\gamma}$ then we can obtain the following equation

$$
\alpha_{1} \frac{\partial f}{\partial x_{1}} x_{1}+\alpha_{2} \frac{\partial f}{\partial x_{2}} x_{2}=f
$$

when $\lambda=1$. and it is traceability that $\alpha_{1}, \alpha_{2} \geq 1$ from the properties of concave function.

Again by substituting $U=\frac{y}{z_{1}}, S=\frac{z_{2}}{z_{1}}, z_{1}=x_{1}^{\frac{1}{\alpha_{1}}}, z_{2}=x_{2}^{\frac{1}{\alpha_{2}}}$, where $z_{1}, z_{2}$ are said to be effective labors, then we get the following equation.

$$
y=f\left(z_{1}, z_{2}\right)=z_{1} \Phi\left(\frac{z_{2}}{z_{1}}\right) \Rightarrow \frac{y}{z_{1}}=\Phi\left(\frac{z_{2}}{z_{1}}\right)
$$

Then the production function has the following general form:

$$
U=\Phi(S)
$$

where $S$ is per labor capita and $U$ is per capita production. By the economic low, $U$ and $S$ s growth velocity rate is defined by the following equation.

$$
\left\{\begin{array}{l}
\dot{S}=n\left(1-\frac{S}{S_{\max }}\right) S=\frac{\mathrm{d} S}{\mathrm{~d} t} \\
\dot{U}=\alpha(n)\left(1-\frac{U}{U_{\max }}\right) U=\frac{\mathrm{d} U}{\mathrm{~d} t} \\
S(0)=S_{0}, \quad U(0)=U_{0}
\end{array}\right.
$$


we can solve the equation then

$$
\left\{\begin{array}{l}
S=\frac{S_{\max } S_{0} \mathrm{e}^{n t}}{\left(S_{\max }-S_{0}\right)+S_{0} \mathrm{e}^{n t}} \\
U=\frac{U_{\max } U_{0} \mathrm{e}^{\alpha(n) t}}{\left(U_{\max }-U_{0}\right)+U_{0} \mathrm{e}^{\alpha(n) t}}
\end{array}\right.
$$

If we express the equation by $S$ then we have

$$
U=\frac{U_{\max } U_{0} \psi\left(\frac{S \cdot \Delta S}{S_{0}\left(S_{\max }-S\right)}\right)}{\left(U_{\max }-U_{0}\right)+U_{0} \psi\left(\frac{S \cdot \Delta S}{S_{0}\left(S_{\max }-S\right)}\right)}
$$

If we choose $\alpha(n)=n \sigma$ then (24) function have the following form:

$$
U=U(S)=\frac{U_{\max } U_{0}\left(\frac{S \cdot \Delta S}{S_{0}\left(S_{\max }-S\right)}\right)^{\sigma}}{\left(U_{\max }-U_{0}\right)+U_{0}\left(\frac{S \cdot \Delta S}{S_{0}\left(S_{\max }-S\right)}\right)^{\sigma}}
$$

We can show that it is a general form of the production function.

\section{For instance}

1) In the Equation (25), If $S_{\max }, U_{\max } \rightarrow \infty$ then

$$
U=U_{0}\left(\frac{S}{S_{0}}\right)^{\sigma}
$$

expressing by $x_{1}, x_{2}, y$, we have

$$
y=A \cdot x_{1}^{\frac{1}{\alpha_{1}}} \cdot\left(\frac{x_{2}^{\frac{1}{\alpha_{2}}}}{x_{1}^{\frac{1}{\alpha_{1}}}}\right)^{\sigma}=A \cdot x_{1}^{\alpha} \cdot x_{2}^{\beta}
$$

(27) is a Cobb-Douglas function (Romer, 1996),

$A=U_{0} \frac{1}{S_{0}^{\sigma}}, \alpha=\frac{1}{\alpha_{1}}(1-\sigma), \beta=\frac{\sigma}{\alpha_{2}}$.

2) Express $\alpha(n)=n \cdot \sigma+\lambda$ then $y=A x_{1}^{\alpha} x_{2}^{\beta} \mathrm{e}^{\lambda t}$.

3) In the Equation (25), $U_{\max } \rightarrow \infty$ and $\alpha(n)=n$ then

$$
U=U_{0}\left(\frac{S \cdot \Delta S}{S_{0}\left(S_{\max }-S\right)}\right)
$$

expressing by $x_{1}, x_{2}, y$ then

$$
y=A x_{1}^{\frac{1}{\alpha_{1}}} \frac{\frac{x_{2}^{\frac{1}{\alpha_{2}}}}{x_{1}^{\frac{1}{\alpha_{1}}}}}{S_{\max }-\frac{x_{2}^{\frac{1}{\alpha_{2}}}}{x_{1}^{\frac{1}{\alpha_{1}}}}}=A \frac{x_{2}^{\frac{1}{\alpha_{2}}}}{S_{\max }-\frac{x_{2}^{\frac{1}{\alpha_{2}}}}{x_{1}^{\frac{1}{\alpha_{1}}}}}=\frac{A}{S_{\max } x_{2}^{-} \frac{1}{\alpha_{2}}-x_{1}^{\frac{1}{\alpha_{1}}}}
$$


$A\left(a x_{1}^{-\frac{1}{\alpha_{1}}}+b x_{2}^{-\frac{1}{\alpha_{2}}}\right)^{-1}$ is a Solow's function.

4) When $\alpha_{1}=\alpha_{2}$ then $y=A\left(a x_{1}^{-\sigma}+b x_{2}^{-\sigma}\right)^{-1}$, it is a function with degree $\sigma$ and constant elasticity.

5) In Equation (25), $S_{\max } \rightarrow \infty$ then

$$
U(S)=U=\frac{U_{\max } U_{0}\left(\frac{S}{S_{0}}\right)^{\sigma}}{\left(U_{\max }-U_{0}\right)+U_{0}\left(\frac{S}{S_{0}}\right)^{\sigma}}
$$

if we express, $A=U_{\max }, a=\frac{U^{\frac{1}{\sigma}}}{S_{0}}, b=U_{\max }-U_{0}$ then

$$
U=U(S)=\frac{A(a S)^{\sigma}}{b+(a S)^{\sigma}}
$$

express by $x_{1}, x_{2}, y$ then

$$
y=\frac{A x_{1}^{\frac{1}{\alpha_{1}}}\left(a \frac{x_{2}^{\frac{1}{\alpha_{2}}}}{S_{0}}\right)^{\sigma}}{b+\left(a \frac{x_{2}^{\frac{1}{\alpha_{2}}}}{x_{1}^{\frac{1}{\alpha_{1}}}}\right)^{\sigma}}
$$

6) The concavity and convexity conditions of the function (31) are

$$
\begin{aligned}
& \frac{\mathrm{d} U}{\mathrm{~d} S}=\frac{A \sigma(a S)^{\sigma-1} a b}{\left(b+(a S)^{\sigma}\right)^{2}} \geq 0, \\
& \frac{\mathrm{d}^{2} U}{\mathrm{~d} S^{2}}=\frac{A(a S)^{\sigma-2} a^{2} b(\sigma-1) b-(\sigma+1)(a S)^{\sigma}}{\left(b+(a S)^{\sigma}\right)^{3}}
\end{aligned}
$$

The function is concave when

$$
\left(\frac{\sigma-1}{\sigma+1} b\right)^{\frac{1}{\sigma}} \leq a S
$$

and quasi-concave when

$$
\left(\frac{\sigma-1}{\sigma+1} b\right)^{\frac{1}{\sigma}}<a S
$$

\section{Numerical Experiments}

In this part, we evaluate and compare the parameters of the quasi homogeneous function and classical production functions using specific economic indicators of Japan which are shown in Table 1. 
Table 1. The main indicators of Japan.

\begin{tabular}{cccccccc}
\hline year & $Y$ & $K$ & $L$ & year & $Y$ & $K$ & $L$ \\
\hline 1955 & 33.2 & 33.8 & 79.9 & 1965 & 100 & 101 & 99 \\
1956 & 38.2 & 37.6 & 84.5 & 1966 & 109.6 & 110.6 & 99.5 \\
1957 & 42.6 & 42.4 & 87.6 & 1967 & 120.3 & 123.9 & 102.2 \\
1958 & 47.4 & 47.0 & 91.4 & 1968 & 131.2 & 138.1 & 106.4 \\
1959 & 53.5 & 52.2 & 95.8 & 1969 & 141.8 & 154.3 & 110.9 \\
1960 & 60.3 & 58.6 & 97.6 & 1970 & 153.4 & 170.4 & 115.7 \\
1961 & 66.3 & 65.6 & 96.3 & 1971 & 167.1 & 186.1 & 118.7 \\
1962 & 79.0 & 72.5 & 96.6 & 1972 & 183.6 & 201.7 & 122.6 \\
1963 & 81.3 & 80.4 & 99.1 & 1973 & 199.9 & 217.7 & 126.6 \\
1964 & 90.6 & 89.7 & 100.7 & 1974 & 215.2 & 235.1 & 130.6 \\
\hline
\end{tabular}

Statistics Bureau of Japan https://www.stat.go.jp/english/ (Statistics Bureau of JAPAN).

Firstly, we consider the Cobb-Duglas function that is

$$
y=A \cdot x_{1}^{\alpha} \cdot x_{2}^{\beta} \cdot \mathrm{e}^{\lambda t}
$$

and the parameters are evaluated as

$$
\begin{array}{ll}
A=0.26261, & \beta=0.13860 \\
\alpha=1.22422, & \gamma=-0.0331
\end{array}
$$

and determination coefficient is

$$
r s=0.0118793
$$

Second, we evaluate the parameters of our new function given by

$$
y=\frac{A x_{1}^{\frac{1}{\alpha_{1}}}\left(a \frac{x_{2}^{\frac{1}{\alpha_{2}}}}{S_{0}}\right)^{\sigma}}{b+\left(a \frac{x_{2}^{\frac{1}{\alpha_{2}}}}{x_{1}^{\frac{1}{\alpha_{1}}}}\right)^{\sigma}} \cdot \mathrm{e}^{\lambda t}
$$

then we have following results

$$
\begin{gathered}
A=3.60827, \quad a=1.53651, \quad \gamma=0.07003 \\
\alpha_{1}=1.46892, \quad b=0.06017, \quad r s=0.00135732 \\
\alpha_{2}=4.460104, \quad \sigma=1.5514
\end{gathered}
$$

\section{Conclusion}

In this paper, we proposed a quasi-homogeneous production function and showed how to construct the production function based on Euler's theorem and the hypothesis that the production function is a quasi-homogeneous and quasi-concave. We also proved that the classical production functions are the special 
cases of the quasi-homogeneous production function. The numerical results show that the quasi-homogeneous function is practically more useful.

\section{Conflicts of Interest}

The authors declare no conflicts of interest regarding the publication of this paper.

\section{References}

Alina-Daniela, V., \& Gabriel-Eduard, V. (2019) On Quasi-Homogeneous Production Functions. Symmetry MDPI Publisher.

Arrow, K. J., \& Enthoven, A. C. (1961) Quasi-Concave Programming. Econometrica, 29, 779-800. https://doi.org/10.2307/1911819

Romer, D. (1996) Advanced Macroeconomics. Berkeley: University of California.

Intriligator, M.D. (2002) Mathematical Optimization and Economic Theory. Moskov: Airis Press. https://doi.org/10.1137/1.9780898719215

Mayer, J. (2007) Quasiconcave Programming.

Solow, R. M. (1956) A Contribution to the Theory of Economic Growth. Quarterly Journal of Economics, 70, 65-94. https://doi.org/10.2307/1884513

Statistics Bureau of Japan. https://www.stat.go.jp/english/ 